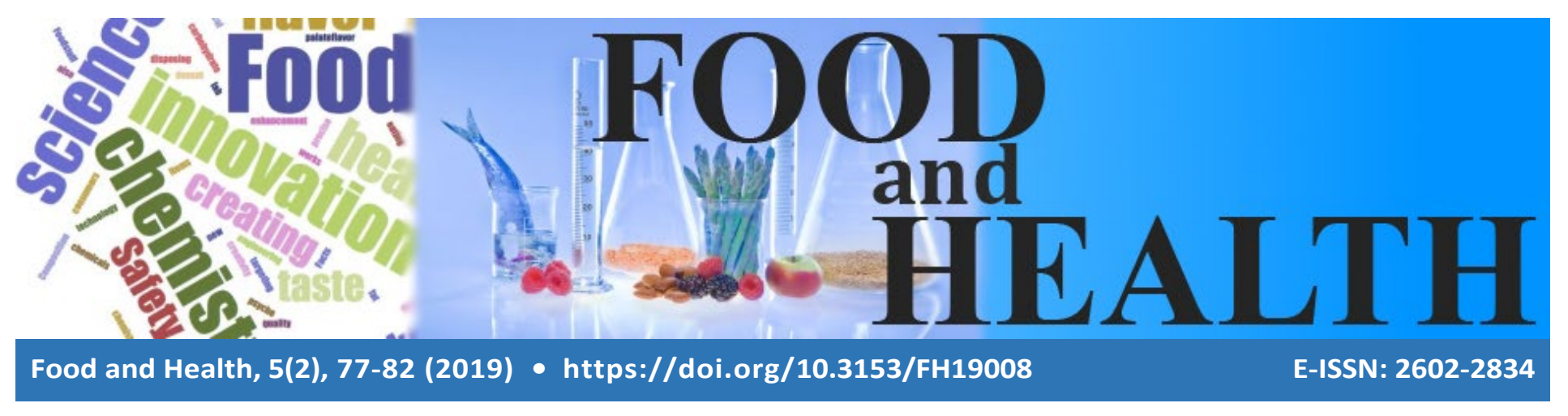

Research Article

\title{
EVALUATING THE PERFORMANCE OF ATP BIOLUMINESCENCE METHOD BY COMPARISON WITH CLASSICAL CULTURAL METHOD
}

\author{
Pinar Öz $\odot$, Özge Özgen Arun $\odot$
}

Cite this article as:

Öz, P., Özgen Arun, Ö. (2019). Evaluating the performance of ATP bioluminescence method by comparison with classical cultural method. Food and Health, 5(2), 77-82. https://doi.org/10.3153/FH19008

Istanbul University Cerrahpaşa Veterinary Faculty Food Hygiene and Technology Department, Avcilar, Istanbul, Turkey

ORCID IDs of the authors: P.Ö. 0000-0002-6474-7061

Ö.Ö.A. 0000-0002-9741-1580

Submitted: 08.03 .2018

Accepted: 30.08 .2018

Published online: 25.10 .2018

Correspondence:

\section{Özge ÖZGEN ARUN}

E-mail: oarun@istanbul.edu.tr

\begin{abstract}
Verification of the performance of the sanitation procedure is as important as the actual application. The methods that are going to be used for such verification needs to be user friendly and rapid resulting to apply the corrective actions promptly. Additionally, these methods should also be reliable besides their fast resulting. ATP bioluminescence method is recommended to be a solution for such procedures for many years. In this present study, our aim was to evaluate the performance of ATP bioluminescence method by comparison with golden standard classical cultural method. For this we performed parallel testing on surfaces which were experimentally contaminated with different types of microorganisms. The results showed that ATP bioluminescence gives more reliable results in surfaces which do not have any physical dirt and are contaminated with high level of microorganisms. The most reliable results were obtained from Salmonella contaminated surfaces.
\end{abstract}

Keywords: ATP bioluminescense, Food hygiene, Hygiene monitoring, Poultry hygiene, Rapid hygiene monitoring
(C) Copyright 2019 by ScientificWebJournals Available online at http://jfhs.scientificwebjournals.com 


\section{Introduction}

The microbiological safety of food products is strongly related to the hygienic properties of the processing environment. In such conditions, applying convenient sanitation methods becomes mandatory for final product safety. Evaluation of the efficiency of these methods is necessary for verification of such processes. As a matter of fact, these verification practises are required in all food safety standards (BRC 2014; ISO 2009; TSE 2006).Therefore, researchers put significant effort on development of rapid and reliable methods to meet the requirement of routine hygiene monitoring in food production.

Although classical cultural methods are the most reliable golden standards, the time consuming process necessary for these methods are not very practical for routine use. The main expectation from such methods are practicability, rapidity and cost efficiency while their reliability is still a major expectation. For this purpose, several alternative rapid methods have been developed (Rosmini at al. 2004). Most well-known samples of these alternative methods are; 3MTM PetrifilmTM, CompactDry, Contact Plate, Dipslide, DEFT, ELISA, PCR ve ATP bioluminescence hygiene monitoring system (Jasson at al., 2010).

ATP bioluminescence is one of these alternative methods and being recommended for online monitoring of the microbiological activity in biological processes since1960s (Chu et al., 2001). The method is based on the reaction between lusiferin-lusiferaz and ATP which transforms the chemical energy to light energy and the measurement of this energy with a illuminometer (Griffith et al., 1997). Because, this method can be performed with a small and practical portable device it has gained a significant application area in food industry for monitoring of production surfaces. With this method it is possible to monitor the level of hygiene in minutes and thus, it gives the possibility of promptly starting the corrective actions if a nonconformity is detected (Chen and Godwin 2006; Chollet et al., 2008; Siragusa et al., 1995).

Several studies have been done so far to evaluate the effectiveness of the method in hygiene monitoring (Ayçiçek et al., 2006; Boyce at al. 2009; Carroscosa et al., 2012; Chen and Godwin 2006; Costa et al., 2006; Davidson et al., 1999; Larson et al., 2003; Lehto et al., 2011; Moore and Griffith 2002; Moore et al., 2010; Sala et al., 2008; Valat et al., 2003). Lehto et al., (2011) used ATP bioluminescence method in a vegetable processing environment in Finland and determined that ATP bioluminescence method can even be used for monitoring of production surfaces with a low contamination level. Similarly, Ayçiçek et al., (2006) compared the results of hygiene monitoring with classical cultural method and ATP at hospital kitchen.

In these studies the evaluation is studied on naturally contaminated surfaces. In contrast, the main purpose of our study was to evaluate the performance of ATP bioluminescence method by comparison with the classical cultural (swab) method on surfaces artificially contaminated with different microorganisms (Salmonella, Echericia coli, Staphylococcus aureus, Saccaromyces cerevisiae). Additionally, we evaluated the performance of the method on naturally contaminated environment. For this, we made parallel sampling with these two methods from a poultry processing plant.

\section{Materials and Methods}

Sampling surfaces and Reference strains: $30 \mathrm{x} 40 \mathrm{~cm}$ stainless steel surfaces which are most commonly used materials in food production were used as model sampling surfaces. Before the artificial contamination, the surfaces were cleaned and disinfected in accordance with a sanitation procedure used by a poultry processing plant in Turkey. For this, the surfaces were sprayed with $1 / 250$ diluted Antec DSC 1000 (Refarm, Istanbul) and rinsed with water for 2-3 min. Following cleaning, the surfaces were sprayed with 1/200 diluted Antec Ambicide (Refarm, Istanbul) for disinfection. Different then the practical approaches, the surfaces were soaked with alcohol and flamed. The residual microorganisms on the surfaces were checked with swab sampling and enumerated on Plate Count Agar (PCA, Oxoid, UK).

For the artificial contamination of the surfaces; lyophilised reference strains of $E$. coli ATCC 25922, Salmonella enterica subsp.enterica serovar Typhi. ATCC 14028, S.aureus subsp. aureus ATCC 11632, S. cerevisiae ATCC 9763 were used. The contamination was performed with stock cultures prepared in compliance with the manufacturer's instructions.

Contamination of the surfaces: a single colony from each reference strain was inoculated in Brain Hearth Infusion Broth (BHIB, Oxoid, UK) and incubated under the appropriate condition and time (at $35^{\circ} \mathrm{C}$ for $24 \mathrm{~h} . ; 44^{\circ} \mathrm{C}$ for $24 \mathrm{~h}$; $35^{\circ} \mathrm{C}$ for $48 \mathrm{~h}$; $25^{\circ} \mathrm{C}$ for 5-7 days; for Salmonella, E. coli, $S$. aureus, $S$. cerevisiae respectively) . The presumptive colony count of these inoculation solutions were determined with McFarland Optical density Equipment (Biosan, 0501021108-0341). The inoculation solution was then 10 fold diluted to prepare the necessary concentration for target contamination level. 
Each of the two parallel surfaces (each for swab and ATP bioluminescence method) were streaked with $0.1 \mathrm{~mL}$ from the appropriate dilution of inoculation solution to give the high $\left(10^{5}-10^{6} \mathrm{cfu} / \mathrm{cm}^{2}\right)$, medium $\left(10^{2}-10^{4} \mathrm{cfu} / \mathrm{cm}^{2}\right)$ and low $\left(10^{1}-10^{2} \mathrm{cfu} / \mathrm{cm}^{2}\right)$ contamination levels. To determine the accurate bacterial count of the inoculation solution, it was plated on PCA (e.g. Plate Count Agar) in duplicate in parallel with the artificial contamination and incubated $48 \mathrm{~h}$ at $35^{\circ} \mathrm{C}$. The obtained enumeration results were used for the determination of the contamination level. This application was repeated for each trial and each reference strain.

ATP measurements: In this study ATP bioluminescence Hygiena (SystemSURE Plus, INS0047) was used according to the instructions manual. Samples were taken from one of the parallel contaminated surface. Sampling were made from $100 \mathrm{~cm}^{2}$ surface. The RLU values read from the instrument was recorded.

Classical cultural method: Swab sampling was performed from the second parallel contaminated surface and the swab stick was left in sterile physiological saline water. Following 10 fold dilutions with sterile physiological saline water, petri dishes containing adequate media were inoculated with appropriate dilution fluid (FDA, 2003; ISO, 2004). For E.coli enumeration TBX agar (Oxoid, UK), for S.aureus Baird Parker Agar (Oxoid, UK), for Salmonella XLD agar (Oxoid, UK) and for total mesophilic aerobic count PCA (Oxoid, UK) were used in accordance with the reference standard methods (FDA, 2001a; FDA 2001b; FDA 2001c; FDA, 2007, ISO 2001).

The whole experimental design was repeated three times.

The correlation between $\log _{10} \mathrm{cfu}$ and RLU values obtained from the artificially contaminated surfaces were calculated with Spearman Correlation method.

\section{Results and Discussion}

The main objective of this study was to evaluate the performance of ATP bioluminescence hygiene monitoring method by comparison with golden standard classical cultural method. For this, sampling from the experimentally contaminated surfaces were analysed with both methods and the results were compared. Additionally, the results of parallel sampling from naturally contaminated processing environment was performed.
The swab sampling taken from the disinfected surfaces did not show any microbiological growth on PCA. This negative total aerobic count showed that the surfaces were successfully decontaminated and the microbial flora we detected after artificial contamination belongs to target microorganisms.

The mean (mean of three repeated experiments) cfu and RLU values for the four contaminated microorganism and all contamination levels are summarized in Table 1.

According to the user's manual of the ATP Bioluminescence equipment (SystemSURE Plus, INS0047) the RLU values $<10,11-29$ and $>30$ reflects clean, conditionally clean and dirty surfaces (Whitehead at al. 2008). The evaluation at the last column of the table were made according to these limits.

The mean RLU results of artificially contaminated surfaces with Salmonella was 189 for high contamination level (6.11 $\log 10 \mathrm{cfu} / \mathrm{cm}^{2}$ ), while 31 and 0 respectively for medium $\left(4.53 \log 10 \mathrm{cfu} / \mathrm{cm}^{2}\right)$ and low $\left(2.29 \log 10 \mathrm{kob} / \mathrm{cm}^{2}\right)$ contamination levels (Table 1). The results for E.coli were 265, 8 and 1 for high $\left(5.57 \log 10 \mathrm{cfu} / \mathrm{cm}^{2}\right)$, medium $(3.25$ $\left.\log 10 \mathrm{cfu} / \mathrm{cm}^{2}\right)$ and low $\left(1.51 \log 10 \mathrm{cfu} / \mathrm{cm}^{2}\right)$ respectively, for S.aureus 136, 19 and 1.6 for high $\left(5.55 \log 10 \mathrm{cfu} / \mathrm{cm}^{2}\right)$, medium (4.46 $\left.\log 10 \mathrm{cfu} / \mathrm{cm}^{2}\right)$ and low $\left(2.95 \log 10 \mathrm{cfu} / \mathrm{cm}^{2}\right)$ respectively and for $S$. cerevisiae 135,0 and 3 for high ( 6.14 $\left.\log 10 \mathrm{cfu} / \mathrm{cm}^{2}\right)$, medium $\left(2.76 \log 10 \mathrm{cfu} / \mathrm{cm}^{2}\right)$ and low $(1.32$ $\left.\log 10 \mathrm{cfu} / \mathrm{cm}^{2}\right)$ respectively. According to these results, ATP bioluminescence evaluated the highly contaminated surfaces which carried 5-6 $\log 10 \mathrm{cfu} / \mathrm{cm} 2$ microorganisms as "dirty". The detection for medium level contaminated surfaces (3-4 $\left.\log 10 \mathrm{cfu} / \mathrm{cm}^{2}\right)$ differed for microorganism and resulted as dirty and doubtful for Salmonella and S.aureus respectively while it resulted as clean for E.coli. The medium level contamination was $2 \log 10 \mathrm{cfu} / \mathrm{cm}^{2}$ for S.cerevisiae and ATP again resulted as clean. The results for low level contamination of S.cerevisiae which is 1-2 $\log 10$ $\mathrm{cfu} / \mathrm{cm}^{2}$ ATP failed to detect or detected as very low. Similarly, Chen and Godwin (2006) also informed that ATP results were lower than 20 RLU for surfaces contaminated with total aerobic bacteria below $3 \log 10 /$ cfu. Likewise, some other researchers calculated the detection limit of ATP 3-4 $\log 10 \mathrm{cfu} / \mathrm{cm}^{2}$ (Siragusa et al., 1995; Davidson et al., 1999; Corbit et al., 2000; Larson et al., 2003; Leon ve Albrech 2007; Sala et al., 2008; Jasson et al., 2010; Carroscosa et al., 2012). 
Table 1. The mean cfu and RLU values for the four contaminated microorganism and all contamination levels

\begin{tabular}{ccccc}
\hline Microorganism & Contamination level & $\mathbf{L o g}_{\mathbf{1 0}} \mathbf{~} \mathbf{c f u} / \mathbf{c m}^{2}$ & RLU & Clean/Dirty \\
\hline \multirow{3}{*}{ Salmonella } & High & 6.11 & 189 & Dirty \\
\cline { 2 - 5 } & Medium & 4.53 & 31 & Dirty \\
\cline { 2 - 5 } & Low & 2.29 & 0 & Clean \\
\hline \multirow{3}{*}{ E.coli } & High & 5.57 & 265 & Dirty \\
\cline { 2 - 5 } & Medium & 3.25 & 8 & Clean \\
\cline { 2 - 5 } & Low & 1.51 & 1 & Clean \\
\hline \multirow{3}{*}{ S.aureus } & High & 5.55 & 136 & Dirty \\
\cline { 2 - 5 } & Medium & 4.46 & 19 & Conditionally clean \\
\cline { 2 - 5 } & Low & 2.95 & 1.6 & Clean \\
\hline \multirow{3}{*}{ S. cerevisiae } & High & 6.14 & 135 & Dirty \\
\cline { 2 - 5 } & Medium & 2.76 & 0 & Clean \\
\cline { 2 - 5 } & Low & 1.32 & 3 & Clean \\
\cline { 2 - 5 }
\end{tabular}

The correlation between the results of classical cultural methods and ATP bioluminescence method was calculated with Spearman correlation test. According to this calculation, correlation coefficient (r) was 0.911, 0.724, 0.644 and 0.623 for Salmonella, E. coli, S. aureus, S. cerevicia respectively. Nevertheless, the correlation coefficient (r) between the classical cultural and ATP bioluminescence method calculated from all results irrespective from the microorganism was $0.761(\mathrm{P}<0.001)$, which indicated that there is a significant correlation between the results. Similar to our results, some other researchers also determined that the correlation between cfu and RLU values differs for different microorganisms and was between 0.68 and 0.90 (Siragusa et al., 1995; Cutter et al., 1996; Chen and Godwin 2006; Sala et al., 2008). In our study, the highest correlation was determined for Salmonella $(\mathrm{r}=0.911)$ while it was as low as $\mathrm{r}=0.623$ for $S$. cerevisciae. The $\mathrm{p}$ values were calculated as $\mathrm{p}=0.073$ and $\mathrm{p}=0.061$ for $S$. cerevisciae and $S$. aureus respectively. Chen and Godwin (2006), also informed that the values determined for different bacteria (total mesophyll aerobic count and psychrophilic count) were different and best results were obtained from mean results of pooled data. This also explains the variations of $(\mathrm{r})$ values obtained from different studies. The results of Moore et al., (2010) on $S$. aureus were in compliance with our results. In their study, the efficiency of the cleaning procedure was inspected in a hospital intensive care service by performing measurements with classical cultural method and ATP bioluminescence method. The results of the study indicated that ATP method resulted as clean while presence of $S$. aureus was confirmed with classical cultural method.

Our results, particularly the results of Salmonella and E.coli is significantly differed from similar studies. Most probably, because we used artificially contaminated surfaces which did not contain any physical dirt. However, in other studies most commonly naturally contaminated surfaces were used and the dirt on these surfaces probably interfered with the results. Similarly, in some other studies the researchers also studied on naturally contaminated surfaces and determined low correlation and finally recommended to perform study with previously disinfected surfaces (Costa et al., 2006; Carroscosa et al., 2012; Vilar et al., 2008). Additionally, Sokolinska ve Pikul (2008) also informed that the surface material is effective on the results and thus this might be another effective factor on the difference of the results.

Our results and results of several other researchers proved that ATP bioluminescence method has a low reliability on naturally contaminated surfaces and does not give specific information about the microorganism and thus it could only be an alternative for monitoring of the sanitation performance on clean surfaces (Davidson et al., 1999; Griffith et al., 2000; Aytaç et al., 2001; Ayçiçek et al., 2006; Chen and Godwin 2006; Costa et al., 2006; Boyce et al., 2009; Jasson et al., 2010; Moore et al., 2010).

\section{Conclusions}

Our results showed that there are several factors effective on the correlation of the results of ATP bioluminescence method and golden standard classical cultural method. However, the meaningful correlation of polled data obtained from ATP irrespective from the microorganism type showed that the method can successfully be used for general verification purposes of the clean surfaces. Additionally because method gave the best correlation with Salmonella and taking it rapidity in to consideration, the method could be recommended for poultry industry as a practical solution. 


\section{Compliance with Ethical Standard}

Conflict of interests: The authors declare that for this article they have no actual, potential or perceived the conflict of interests.

Financial disclosure: This Project was supported by Research Fund of Istanbul University, Project No: 14970

\section{References}

Ayçiçek, H., Oğuz, U., Karc1, K. (2006). Comparison of results of ATP bioluminescence and traditional hygiene swabbing methods for the determination of surface cleanliness at a hospital kitchen. International Journal of Hygiene and Environmental Health, 209(2), 203206.

Aytaç, S.A., Mercanoğlu, B., Özbaş, Y. (2001): Enumeration of Escherichia coli $0157: \mathrm{H} 7$ by using immunomagnetic separation and ATP bioluminescence in buffer solution. Turkish Hygiene and Experimental Biology Journal, 58(2), 49-52.

Boyce, J.M., Havill, N.L., Dumigan, D.G., Golebiewski, M., Balogun, O., Rizvani, R. (2009). Monitoring the effectiveness of hospital cleaning practices by use of an adenosine triphosphate bioluminescense assay. Infection Control and Hospital Epidemiology, 30(7), 678684.

BRC (2014). Global standard for food safety. Issue 7. British Retail Consortium, UK.

Carroscosa, C., Saavedra, P., Millán, R., Jaber, J. R., Pérez, E., Grau, R., Raposa, A., Mauricio, C., Sanjuan, A. (2012). Monitoring of cleanliness and disinfection in dairies: Comparison of traditional microbiological and ATP bioluminescence methods. Food Control, 28, 368-373.

Chen F.C., Godwin, S.L, (2006). Comparison of a rapid ATP bioluminescence assay and standard plate count methods for assessing microbial contamination of consumers refrigerators. Journal of Food Protection, 69(10), 2534-2538.

Chollet, R., Kukuczka, M., Halter, N., Romieux, M., Marc, H.M., Benguin, V., Raibault, S. (2008). Rapid detection and enumeration of contaminants by ATP bioluminescense using the milliflex rapid microbiology detection and enumeration system. Journal of Rapid Methods and Automation in Microbiology, 16, 256-272.
Chu, C.P., Lee, D. J., Chang, B.V., Liao, C. S. (2001). Using ATP bioluminescence technique for monitoring microbial activity in sludge. Biotechnology and Bioengineering, 75(4), 469-474.

Corbit, A.J., Bennion, N., Forsythe, S.J. (2000). Adenylate kinase amplification of ATP bioluminescence for hygiene monitoring in the food and beverage industry. Letters in Applied Microbiology, 30(6), 443-447.

Costa, P.D., Andrade, N.J., Brandao, S.C.C., Passos, F.J.V., Soares, N.F.F. (2006). ATP-Bioluminescence assay as an alternative for hygiene-monitoring procedures of stainless steel milk contact surfaces. Brazilian Journal of Microbiology, 37, 345-349.

Cutter. C.N., Dorsa, W.J., Siragusa, G.R. (1996). A rapid microbial ATP bioluminescence assay for meat carcasses. Dairy Food Environment, 66, 726-736.

Davidson, C.A., Griffith, C.J., Peters, A.C., Fielding, L.M. (1999). Evaluation of two methods for monitoring surface cleanliness - ATP bioluminescence and traditional hygiene swabbing. Luminescence, 14(1), 33-38.

FDA. (2001a). Food and Drug Administration. Chapter 3. Aerobic Plate Count. Bacteriological Analytical Manual. Retrieved from:

https://www.fda.gov/Food/FoodScienceResearch/LaboratoryMethods/ucm063346.htm (05.03.2018)

FDA. (2001b). Food and Drug Administration. Chapter 12. Staphylococcus aureus. Bacteriological Analytical Manual. Retrieved from:

https://www.fda.gov/Food/FoodScienceResearch/LaboratoryMethods/ucm071429.htm (05.03.2018)

FDA. (2001c). Food and Drug Administration. Chapter 18. Yeast, Moulds and Mycotoxins. Bacteriological Analytical Manual. Retrieved from: https://www.fda.gov/Food/FoodScienceResearch/LaboratoryMethods/ucm071435.htm (05.03.2018)

FDA. (2003). Food Sampling and Preparation of Sample Homogenate Chapter 1 Bacteriological Analytical Manual. Retrieved from: https://www.fda.gov/Food/FoodScienceResearch/LaboratoryMethods/ucm063335.htm (05.03.2018) 
FDA. (2007). Food and Drug Administration. Chapter 5. Salmonella Bacteriological Analytical Manual. Retrieved from: https://www.fda.gov/Food/FoodScienceResearch/LaboratoryMethods/ucm070149.htm (05.03.2018)

Griffith, C.J., Davidson, C.A., Peters, A.C., Fielding, L.M. (1997). Towards a strategic cleaning assessment programme: hygiene monitoring and ATP luminometry, an options appraisal, Food Science and Technology, 11, $15-24$.

Griffith, C.J., Cooper, R.A., Gilmore, J., Davies, C., Lewis, M. (2000): An evaluation of hospital cleaning regimes and standards. Journal of Hospital Infection, 45, 19-28.

ISO (2001). Microbiology of food and animal feeding stuffsHorizontal method for the enumeration of beta-glucuronidase-positive E. coli. Part 2: Colony-count technique at 44 degrees $C$ using 5-bromo-4-chloro-3-indolyl beta-D-glucuronide, 16649. International Standard Organisation, Geneva, Switzerland.

ISO (2004). Microbiology of food and animal feeding stuffs-Horizontal methods for sampling techniques from surfaces using contact plates and swabs, ISO 18593. International Standard Organisation, Geneva, Switzerland.

ISO (2009). Prerequisite programmes on food safety. Part 1: Food manufacturing, ISO 22002-1. International Standard Organisation, Geneva, Switzerland.

Jasson, V., Jacxsens, L., Luning, P., Rajkovic, A., Uytendaele, M. (2010). Alternative microbial methods: An overview and selection criteria. Food Microbiology, 27, 710-730.

Larson, L. E., Ailello, A.E., Gomez-Duarte, C., Lin, S.X., Lee, L., Della-Latta, P., Lindhardt, C. (2003). Bioluminescense ATP monitoring as a surrogate marker for microbial load on hands and surfaces in the home. Food Microbiology, 20, 735-739.

Lehto, M., Kuisma, R., Määttä, J., Kymäläinen, H.R., Mäki, M. (2011). Hygienic level and surface contamination in fresh-cut vegetable production plants. Food Control, 22, 469-475.

Leon, M.B., Albrecht, J.A. (2007). Comparison of adenosine triphosphate (ATP) bioluminescence and aerobic plate counts (APC) on plastic cutting boards. Journal of Food Service, 18, 145-152.

Moore, G., Griffith, C. (2002). A comparison of surface sampling methods for detecting coliforms on food contact surfaces. Food Microbiology, 19, 65-73.

Moore, G., Smyth, D., Singleton, J., Wilson, P. (2010). The use of adenosine triphosphate bioluminescense to assess the efficacy of a modified cleaning program implemented within an intensive care setting. American Journal of Infection Control, 38, 617-622.

Rosmini, M.R., Signorini, M.L., Schneider, R., Bonazza, J.C., (2004). Evaluation of two alternative techniques for counting mesophilic aerobic bacteria in raw milk. Food Control, 15, 39-44.

Sala, C., Milovan, G.H., Morar, A., Niichita, I. (2008). Establishing the microbial contamination of surtaces from the food industry by alternative methods using ATP bioluminescense. Lucrăr Stiintifice Medicină Veterinară, 41, 841-846.

Siragusa, G.R., Cutter, C.N., Dorsa, W.J., Koohmaraie, M. (1995). Use of a rapid microbial ATP bioluminescence assay to detect contamination on beef and pork carcasses. Journal of Food Protection, 58(7), 770-775.

Sokolinkska, C.D. and Pikul, J. (2008). Evalution of steel surface cleanliness level in dairies using the bioluminescense method. Bulletin of the Veterinary Institute in Pulawy, 52, 625-629.

TSE (2006). Food Safety management systems. TS EN ISO 22000. Turkish Standard Institute, Ankara.

Valat, C., Champiat, D., N'Guyen, T.T.T., Loiseau, G., Raimbault, M., Montet, D. (2003). Use of ATP bioluminescense to determine the bacterial sensitivity threshold to a bacteriocin. Luminescense, 18, 254-258.

Vilar, M.J., Rodríguez-Otero, J. L., Diéguez, F.J., Sanjuán, M.L., Yus, E. (2008). Application of ATP bioluminescence for evaluation of surface cleanliness of milking equipment. International Journal of Food Microbiology, 125(3), 357-361.

Whitehead, K.A., Smith, L., Verran, J. (2008). The detection of food soils and cells on stainless steel using industrial methods: UV illumination and ATP bioluminescence. International Journal of Food Microbiology, 127, 121128. 